\title{
Almost anti-periodic solution of inertial neural networks model on time scales
}

\author{
Adnène Arbi ${ }^{1,2, *}$, and Najeh Tahri ${ }^{2}$ \\ ${ }^{1}$ National School of Advanced Sciences and Technologies of Borj Cedria, University of Carthage, \\ Tunisia \\ ${ }^{2}$ Laboratory of Engineering Mathematics (LR01ES13), Tunisia Polytechnic School, University of \\ Carthage, Tunisia
}

\begin{abstract}
In this work, since the importance of investigation of oscillators solutions, an methodology for proving the existence and stability of almost anti-periodic solutions of inertial neural networks model on time scales are discussed. By developing an approach based on differential inequality techniques coupled with Lyapunov function method. A numerical example is given for illustration.
\end{abstract}

Keywords: Dynamical systems, Time scales, Exponential stability, Almost-anti periodic solution, Inertial Neural Networks.

\section{Introduction}

In these ten years, neural networks (NNs) have been successfully applied to numerous fields such as synchronization, control, stability and stabilization of periodic and automorphic solutions ([1], [2], [3],[4], [5], [6],[7], [8], [9], [10], [11], [17]). The aim of this work is to introduce the concept of almost anti-periodic functionson time scales. Then we look for a solution of almost anti-periodic type for dynamic systems modeling Inertial Neural Networks (INNs). The (INNs) model represent a class of artificial neural networks, introduced by Wheeler and Schieve [12] in 1997. Mathematically, it's a second order dynamic system, the term derived from the first order describes the inertial term which naturally covers many fields of application: biology, physics, artificial intelligence. On the other hand, the choice of time scales justified, indeed the theory of time scales is developed by Hilger S., which made it possible to treat continuous-timeand discrete-time equations simultaneously. A time scale is an arbitrary non empty closed subset of $\mathrm{R}$ denoted by $\mathrm{T}$. Hilger notably defined the $\Delta$-derivative as follows:

The function $\mathrm{f}: \mathrm{T} \rightarrow \mathrm{R}$ is said to possess a $\Delta$-derivative $\mathrm{f} \Delta$ if there exists $\varepsilon>0$ such that

$$
\left\|[f(\sigma(t))-f(s)]-f^{\Delta}(t)[\sigma(t)-s]\right\| \leq \varepsilon\|\sigma(t)-s\| \text {, for all } s \in T^{\kappa} .
$$

For more details, the books $[13,14]$ summarize much of time scale calculus.

\footnotetext{
* Corresponding author: adnen.arbi@enseignant.edunet.tn
} 
The innovation of this work is essentially reflected in three parts. First, application to the class of almost anti-periodic functions, this kind of function introduced in literature. Second, we generalize this concept on time scales. Finally, we will give a sufficient condition guarantees the stability which is based on the construction of a Lyapunov function associated with the system (1).

\section{Assumptions and main results}

We start by defining the notion of almost anti-periodic functions on time scales.

Definition $2.1 \mathrm{~T}$ is called periodic time scale if there exists $\theta>0$ such that if $\mathrm{t} \in \mathrm{T}$ then $\theta \pm \mathrm{t} \in \mathrm{T}$.

Definition 2.2 Let $\varepsilon>0$, we call $\theta>0$ an $\varepsilon$-antiperiod for $\varphi$ if $\|\varphi(t+\theta)+\varphi(t)\| \leq \varepsilon, t \in T$. We denote the set of all $\varepsilon$-antiperiods for $\varphi$ by $\operatorname{Vap}(\varphi, \varepsilon)$. It is sait that $\varphi$ is almost anti-periodic if for each $\varepsilon>0$ the set $\operatorname{Vap}(f, \varepsilon)$ is relativety dense in $T$.

In this work, we consider almost-anti-periodic solutions of the following models for inertial neural networks with varying delays on time scales

$$
\begin{gathered}
x_{\mathrm{i}}^{\Delta \Delta}(t)=-a_{\mathrm{i}}(t) x_{\mathrm{i}}^{\Delta}(t)-b_{\mathrm{i}}(t) x_{\mathrm{i}}(t)+\sum_{j=1}^{n} c_{\mathrm{ij}}(t) f_{j}\left(x_{\mathrm{j}}(t)\right)+\sum_{j=1}^{n} d_{\mathrm{ij}}(t) g_{j}\left(x_{\mathrm{j}}(t)\right)+S_{\mathrm{i}}(t) \\
x_{\mathrm{i}}(s)=\Psi(s), \quad x_{\mathrm{i}}^{\Delta}(s)=¥(s)
\end{gathered}
$$

We assume for all $\mathrm{t}, \mathrm{u}, \mathrm{v} \in \mathrm{T}$ and $\mathrm{i}, \mathrm{j} \in[1 \cdots \mathrm{n}]$

(P1) For $f_{j}$ and $g_{j}$ are all non-decreasing functions with $f_{j}(0)=g_{j}(0)=0$, and there exists $L_{j}^{f}, L_{j}^{g}>0$

such that

$$
\begin{aligned}
\left|f_{j}(u)-f_{j}(v)\right| \leq L_{j}^{f}|u-v|, c_{\mathrm{ij}}(t+w) f_{j}(t) & =-c_{\mathrm{ij}}(t) f_{j}(-t) \\
\left|g_{j}(u)-g_{j}(v)\right| \leq L_{j}^{g}|u-v|, d_{\mathrm{ij}}(t+w) g_{j}(t) & =-c_{\mathrm{ij}}(t) g_{j}(-t)
\end{aligned}
$$

Remark 1 The property (P1) states a sufficient condition to obtain existence and uniqueness of solution of the system (1). This result is a consequence of a Picard-Lindelof theorem of local uniqueness and existence of first-order systems of nonlinear delay dynamic equations on time scales [15], to delve deeper, you can see [16].

(P2) There exists $\alpha_{\mathrm{i}} \geq 0, \gamma_{\mathrm{i}} \geq 0$ and $\beta_{\mathrm{i}}>0$ satisfying

$$
E_{\mathbf{i}}(t)<0,4 E_{\mathbf{i}}(t) F_{\mathbf{i}}(t)>G_{\mathbf{i}}(t), \quad t \in \mathbb{T} \cap \mathbb{R}^{+}
$$

where

$$
\begin{gathered}
E_{\mathrm{i}}(t)=\alpha_{\mathrm{i}} \gamma_{\mathrm{i}}-\alpha_{\mathrm{i}}^{2} a_{\mathrm{i}}(t)+\frac{1}{2} \alpha_{\mathrm{i}}^{2} \sum_{j=1}^{n}\left(\left|c_{\mathrm{ij}}(t)\right| L_{j}^{f}+\left|d_{\mathrm{ij}}(t)\right| L_{j}^{g}\right) \\
F_{\mathrm{i}}(t)=-b_{\mathrm{i}}(t) \alpha_{\mathrm{i}} \gamma_{\mathrm{i}}+\frac{1}{2} \sum_{j=1}^{n}\left(\left|c_{\mathrm{ij}}(t)\right| L_{j}^{f}+\right. \\
\left.\left|d_{\mathrm{ij}}(t)\right| L_{j}^{g}\right)\left|\alpha_{\mathrm{i}} \gamma_{\mathrm{i}}\right| \\
\frac{1}{2} \sum_{j=1}^{n} \alpha_{\mathrm{j}}^{2}\left(\left|c_{\mathrm{ij}}(t)\right| L_{j}^{f}+\left|d_{\mathrm{ij}}^{+}(t)\right| L_{j}^{g}\right)+\frac{1}{2} \sum_{j=1}^{n}\left(\left|c_{\mathrm{ij}}(t)\right| L_{j}^{f}+\right. \\
\left.\left|d_{\mathrm{ij}}^{+}(t)\right| L_{j}^{g}\right)\left|\alpha_{\mathrm{i}} \gamma_{\mathrm{i}}\right|
\end{gathered}
$$




$$
G_{\mathrm{i}}(t)=\beta_{\mathrm{i}}+\gamma_{\mathrm{i}}^{2}-\alpha_{\mathrm{i}} \gamma_{\mathrm{i}} a_{\mathrm{i}}(t)-\alpha_{\mathrm{i}}^{2} b_{\mathrm{i}}(t)
$$

( $\left.\mathrm{P}_{3}\right) \quad$ (a) $a_{\mathrm{i}}, b_{\mathrm{i}}, c_{\mathrm{ij}}$ and $d_{\mathrm{ij}}$ periodic functions.

(b) $\Psi(s), ¥(s)$ and $S_{\mathrm{i}}$ almost anti-periodic functions.

$\left(\mathrm{P}_{4}\right)$ There exists $\lambda>0$ such that

$$
\begin{gathered}
E_{\mathrm{i}}^{\lambda}(t)<0,4 E_{\mathrm{i}}^{\lambda}(t) F_{\mathrm{i}}^{\lambda}(t)>\left(G_{\mathrm{i}}^{\lambda}(t)\right)^{2}, t \in \mathbb{T} \cap \mathbb{R}^{+} \\
E_{\mathrm{i}}^{\lambda}(t)=\alpha_{\mathrm{i}}^{2}+\alpha_{\mathrm{i}} \gamma_{\mathrm{i}}-\alpha_{\mathrm{i}}^{2} a_{\mathrm{i}}(t)+\frac{1}{2} \alpha_{\mathrm{i}}^{2} \sum_{j=1}^{n}\left(\left|c_{\mathrm{ij}}(t)\right| L_{j}^{f}+\left|d_{\mathrm{ij}}(t)\right| L_{j}^{g}\right) \\
F_{\mathrm{i}}^{\lambda}(t)=\lambda \beta_{\mathrm{i}}+\lambda \gamma_{\mathrm{i}}^{2}-b_{\mathrm{i}}(t) \alpha_{\mathrm{i}} \gamma_{\mathrm{i}}+\frac{1}{2} \sum_{j=1}^{n}\left(\left|c_{\mathrm{ij}}(t)\right| L_{j}^{f}\right. \\
\left.+\left|d_{\mathrm{ij}}(t)\right| L_{j}^{g}\right)\left|\alpha_{\mathrm{i}} \gamma_{\mathrm{i}}\right| \\
+\frac{1}{2} \sum_{j=1}^{n} \alpha_{\mathrm{j}}^{2}\left(\left|c_{\mathrm{ij}}(t)\right| L_{j}^{f}+\left|d_{\mathrm{ij}}^{+}(t)\right| L_{j}^{g} e^{2 \lambda}\right)+\frac{1}{2} \sum_{j=1}^{n}\left(\left|c_{\mathrm{ij}}(t)\right| L_{j}^{f}\right. \\
\left.+\left|d_{\mathrm{ij}}^{+}(t)\right| L_{j}^{g} e^{2 \lambda}\right)\left|\alpha_{\mathrm{i}} \gamma_{\mathrm{i}}\right| \\
G_{\mathrm{i}}^{\lambda}(t)=2 \lambda \alpha_{\mathrm{i}} \gamma_{\mathrm{i}}+\beta_{\mathrm{i}}+\gamma_{\mathrm{i}}{ }^{2}-\alpha_{\mathrm{i}} \gamma_{\mathrm{i}} a_{\mathrm{i}}(t)-\alpha_{\mathrm{i}}^{2} b_{\mathrm{i}}(t)
\end{gathered}
$$

Definition 2.3 The system (1) is said to be exponentially stable, if for $\mathrm{x}(\mathrm{t})$ and $\mathrm{y}(\mathrm{t})$ two solutions of $\quad$ system (1) of initial value $x_{\mathrm{i}}(s)=\Psi^{x}(s), x_{\mathrm{i}}^{\Delta}(s)=¥^{x}(s), y_{\mathrm{i}}(s)=\Psi^{y}(s)$, $y_{\mathrm{i}}^{\Delta}(s)=¥^{y}(s)$, there exists $\lambda>0$ and $\mathrm{M}>0$ such that for all $t \in \mathbb{T} \cap \mathbb{R}^{+}$,

$$
\begin{aligned}
\left|x_{\mathrm{i}}(t)-y_{\mathrm{i}}(t)\right| \leq & M\left|\Psi_{\mathrm{i}}^{x}-\Psi_{\mathrm{i}}^{y}\right| e_{-\lambda}(t, s),\left|\Psi_{\mathrm{i}}^{x}-\Psi_{\mathrm{i}}^{y}\right| \\
& =\max _{1 \leq \mathrm{i} \leq \mathrm{n}}\left\{\sup _{-\tau \leq s<0} \Psi_{\mathrm{i}}^{x}(s)-\Psi_{\mathrm{i}}^{y}(s)\right\} \\
\left|x_{\mathrm{i}}{ }^{\Delta}(t)-y_{\mathrm{i}}{ }^{\Delta}(t)\right| & \leq M\left|¥_{\mathrm{i}}{ }^{x}-¥_{\mathrm{i}}{ }^{y}\right| e_{-\lambda}(t, s),\left|¥_{\mathrm{i}}{ }^{x}-¥_{\mathrm{i}}{ }^{y}\right| \\
& =\max _{1 \leq \mathrm{i} \leq \mathrm{n}}\left\{\sup _{-\tau \leq s<0} ¥_{\mathrm{i}}^{x}(s)-¥_{\mathrm{i}}^{y}(s)\right\}
\end{aligned}
$$

Theorem 2.4 Under assumptions (P1)-(P4), the system (1) possesses a globally generalized exponentially stable solution.

Proof.

Step 1: Prove that V is a Lyapunov candidate function associated with system (1) where 


$$
\begin{aligned}
& V(t) \\
& =\frac{1}{2} \sum_{j=1}^{n}\left[\beta_{\mathrm{i}} \mathrm{w}_{\mathrm{i}}^{2}(t)\right. \\
& +\left(\alpha_{\mathrm{i}} w_{\mathrm{i}}^{\Delta}(t)\right. \\
& \left.\left.+\gamma_{\mathrm{i}} w_{\mathrm{i}}(t)\right)^{2}\right] e_{-2 \lambda}(t, s) \\
& +\frac{1}{2} \sum_{\mathrm{i}, \mathrm{j}=1}^{n}\left[\alpha_{\mathrm{i}}^{2}+\left|\alpha_{\mathrm{i}} \gamma_{\mathrm{i}}\right|\right] d_{\mathrm{ij}}^{+} L_{j}^{g} e^{2 \lambda} \int_{0}^{t} w_{j}^{2}(s) e_{-2 \lambda}(t, s) d s
\end{aligned}
$$

Step 2: Try to write the upper right Dini derivative of $\mathrm{V}$ of the following form

$$
\begin{array}{r}
D V^{+}(t) \leq e_{-2 \lambda}(t, s)\left\{\sum_{\mathrm{i}=1}^{n} E_{\mathrm{i}}^{\lambda}(t)\left(w_{\mathrm{i}}^{\Delta+}(t)+\frac{G_{\mathrm{i}}^{\lambda}(t)}{2 E_{\mathrm{i}}^{\lambda}(t)} w_{\mathrm{i}}^{+}(t)\right)^{2}\right. \\
\left.+\sum_{\mathrm{i}=1}^{n} \frac{4 E_{\mathrm{i}}^{\lambda}(t) F_{\mathrm{i}}^{\lambda}(t)-\left(G_{\mathrm{i}}^{\lambda}(t)\right)^{2}}{4 E_{\mathrm{i}}^{\lambda}(t)}\left(w_{\mathrm{i}}^{+}(t)\right)^{2}\right\}
\end{array}
$$

Step 3: Deduce using the property (P2) and (P4) that $D V^{+}(t) \leq 0$.

Remark 2 The advantage of the theory of time scales is to unify continuous and discretetime and to provide a powerful tool for many applications.

\section{Numerical example}

Place ourselves in the case where $\mathbb{T}=\frac{1}{4} \mathbb{Z}, 1 \leq \mathrm{i}, j \leq 2$

$$
\left\{\begin{array}{c}
x_{1}^{\Delta \Delta}(t)=-\frac{30}{9+|\cos (3 t)|} x_{1}^{\Delta}(t)-\frac{28}{9+|\cos (3 t)|} x_{1}(t)+0.72|\cos (3 t)| f_{1}\left(x_{1}(t)\right. \\
+0.65|\cos (3 t)| f_{2}\left(x_{2}(t)\right)+0.53|\cos (3 t)| g_{1}\left(x_{1}(t)\right)+0.32|\cos (3 t)| g_{2}\left(x_{2}(t)\right)+\cos (3 t)
\end{array}\right.
$$

$$
\left\{\begin{array}{c}
x_{2}^{\Delta \Delta}(t)=-\frac{27}{9+|\sin (3 \mathrm{t})|} x_{2}^{\Delta}(t)-\frac{36}{9+|\sin (3 \mathrm{t})|} x_{2}(t)+0.65|\sin (3 \mathrm{t})| f_{1}\left(x_{1}(t)\right. \\
+0.42|\sin (3 \mathrm{t})| f_{2}\left(x_{2}(t)\right)+0.53|\sin (3 \mathrm{t})| g_{1}\left(x_{1}(t)\right)+0.66|\sin (3 \mathrm{t})| g_{2}\left(x_{2}(t)\right)+\sin (3 \mathrm{t})
\end{array}\right.
$$

where $f_{j}(x)=g_{j}(x)=0.5 \arctan (x), L_{j}^{f}=L_{j}^{g}=0.5$ and from $\alpha_{\mathrm{i}}=\gamma_{\mathrm{i}}=1, \beta_{\mathrm{i}}=$ 5.5, $\lambda=0.01$,

the system (4) satisfy all assumptions:

$$
\begin{aligned}
4 E_{1}(t) F_{1}(t) & \geq 3>\left(G_{1}(t)\right)^{2} \\
4 E_{2}(t) F_{2}(t) & \geq 3>\left(G_{2}(t)\right)^{2} \\
4 E_{1}^{\lambda}(t) F_{1}^{\lambda}(t) & \geq 7>\left(G_{1}^{\lambda}(t)\right)^{2} \\
4 E_{2}^{\lambda}(t) F_{2}^{\lambda}(t) & \geq 7>\left(G_{2}^{\lambda}(t)\right)^{2}
\end{aligned}
$$

Theorem 2.4 implies that unique almost anti-periodic solution of system (1) is exponentially stable. We virtue the following transformations in (4) 


$$
(\mathrm{xi} \Delta(\mathrm{t}), \mathrm{xi} \Delta \Delta(\mathrm{t}))=\left(\mathrm{y}_{\mathrm{i}}(\mathrm{t}), \mathrm{yi} \Delta(\mathrm{t})\right)
$$

From (5), the system (4) take the form of first order:

$$
\begin{gathered}
\left\{\begin{array}{c}
x_{1}^{\Delta}(t)=y_{1}(t) \\
y_{1}^{\Delta}(t)=-\frac{30}{9+|\cos (3 t)|} y_{1}(t)-\frac{28}{9+|\cos (3 t)|} x_{1}(t)+0.72|\cos (3 t)| f_{1}\left(x_{1}(t)\right. \\
+0.65|\cos (3 t)| f_{2}\left(x_{2}(t)\right)+0.53|\cos (3 t)| g_{1}\left(x_{1}(t)\right)+0.32|\cos (3 t)| g_{2}\left(x_{2}(t)\right)+\cos (3 t)
\end{array}\right. \\
\left\{\begin{array}{c}
x_{2}^{\Delta}(t)=y_{2}(t) \\
x_{2}^{\Delta \Delta}(t)=-\frac{27}{9+|\sin (3 \mathrm{t})|} y_{2}(t)-\frac{36}{9+|\sin (3 \mathrm{t})|} x_{2}(t)+0.65|\sin (3 \mathrm{t})| f_{1}\left(x_{1}(t)\right. \\
+0.42|\sin (3 \mathrm{t})| f_{2}\left(x_{2}(t)\right)+0.53|\sin (3 \mathrm{t})| g_{1}\left(x_{1}(t)\right)+0.66|\sin (3 \mathrm{t})| g_{2}\left(x_{2}(t)\right)+\sin (3 \mathrm{t})
\end{array}\right.
\end{gathered}
$$

The initial conditions of system (6) are given by folowing almost anti-periodic functions $\mathrm{x}_{1}(\mathrm{~s})=-\sin (\mathrm{s}), \mathrm{y}_{1}(\mathrm{~s})=\sin (\mathrm{s}), \mathrm{x}_{2}(\mathrm{~s})=\sin (\mathrm{s}), \mathrm{y}_{2}(\mathrm{~s})=\sin (\mathrm{s}) \mathrm{s} \in[-0.11,0]_{\mathrm{T}}$
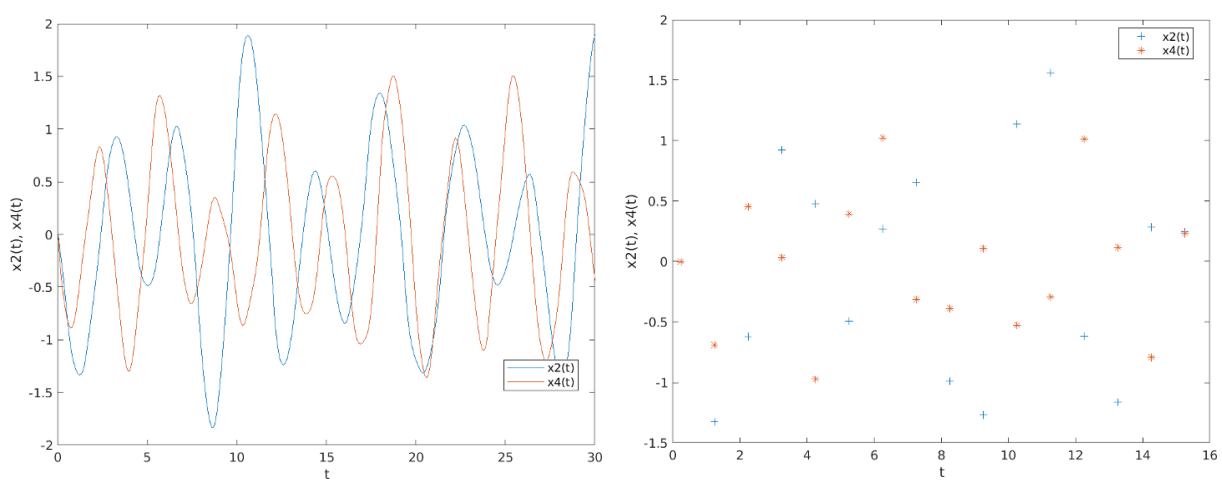

Fig. 3. This analyse proposed in this work, can be extended for a more complex delayed neuronalmodels as example [2] and [0]. These ideas can be the subjects of some future works.

\section{References}

1. ARBI A., CAO J., Alsaedi A.," Improved synchronization analysis of competitive neural networks with time-varying delays", Nonlinear Anal Model Control, 23(1), pp. 82-102, (2018).

2. Kao, Y., Ming, Q., Gao, C., Exponential Stability of Periodic Solutions for CohenGrossberg Neural Networks with Continuously Distributed Delays.Advances in Intelligent Systems Research (2017).

3. Hui, F. Positive periodic solutions of n-species neutral delay systems. Czechoslovak Mathematical Journal 53.3 (2003): 561-570.

4. Yong, R., Yin, W., Zhu, D. Exponential stability of SDEs driven by G-Brownian motion with delayed impulsive effects: average impulsive interval approach. Discrete \& Continuous Dynamical Systems-B 23.8 (2018): 3347.

5. Zahra, A. On synchronous behavior in complex nonlinear dynamical systems. Diss. Rutgers University-Graduate School- New Brunswick, 2015.

6. Shiping, S., Li, B., Li, Y. Anti-periodic dynamics of quaternion-valued fuzzy cellular neural networks with time-varying delays on time scales. Discrete Dynamics in Nature and Society 2018 (2018). 
7. Li, L., Wang, Z., Lu, J., Li, Y. Adaptive synchronization of fractional-order complexvalued neural networks with discrete and distributed delays. Entropy 20.2 (2018): 124.

8. Yongkun, L., Xiang, J., Li, B. Globally asymptotic almost automorphic synchronization of Clifford-valued RNNs with delays. IEEE Access 7 (2019): 54946-54957.

9. Ruoyu, W., Cao, J., Kurths, J. Novel fixed-time stabilization of quaternion-valued BAMNNs with disturbances and time-varying coefficients [J]. AIMS Mathematics 5.4 (2020): 3089-3110.

10. Tingwen, H., Chen, G., Kurths, J., Synchronization of chaotic systems with timevarying coupling delays. Discrete \& Continuous Dynamical Systems-B 16.4 (2011): 1071.

11. Manifolds for Reaction-Diffusion Delayed Neural Networks of Cohen-Grrossberg-Type under Variable Impulsive Perturbations. Mathematics 8.7 (2020): 1082.

12. Wheeler D. \& Schieve W. (1997). Stability and chaos in an inertial two-neuron system. Phys. D. Nonlinear Phenom. 105, 267-284.

13. Bohner M. \& Peterson A. (2001). Dynamic Equations on Time Scales: An introduction with applications. Birkhäuser: Basel.

14. Anatoly A. Martynyuk (2016). Stability Theory for Dynamic Equations on Time Scales, Birkhäuser, Switzerland.

15. Karpuz B. (2011). Existence and uniqueness of solutions to systems of delay dynamic equations on time scales. Int. J. Math. Comput., vol. 10, no. M11, pp. 48-58.

16. Hale J.K (1977). Theory of Functional Differential Equations. Springer New York.

17. Arbi A. (2018). Dynamics of BAM neural networks with mixed delays and leakage time varying delays in the weighted pseudo almost periodic on time space scales. Mathematical Methods in the Applied Sciences,41(3),1230-1255. 